\title{
New approach for non-invasive temperature measurement in process industry
}

\author{
Jörg Gebhardt', Andreas Decker ${ }^{1}$, Paul Szasz ${ }^{1}$, Stephan Wildermuth ${ }^{1}$, \\ Tilo Merlin', Horst Schwanzer2, \\ ${ }^{1}$ ABB Corporate Research Center Germany, Ladenburg, Germany \\ ${ }^{2}$ ABB Process Automation, Measurement and Analytics, Frankfurt / Alzenau, Germany
}

\begin{abstract}
The majority of measurements made in process industry are of temperature and pressure. Temperature measurements are used in particular to secure product quality, increase process efficiency and ensure plant safety. Suitable temperature measurement instruments are widely available and the cost of these has decreased over time due to high volumes, technological progress and competition. However, these devices are mostly intrusive in nature. Noninvasive, wireless and energy-autonomous temperature sensors have the potential to substantially change the practice of industrial temperature sensing. In this article, a successful implementation for such an instrument is provided, covering an optimized, experimentally validated thermomechanical design, a model-based correction accounting for inherent measurement errors, and a pilot installation at an end customer plant.
\end{abstract}

Keywords: Temperature measurement, non-invasive, thermowells, model-based sensing.

\section{Significance and problems of industrial temperature measurement}

The main design of an industrial temperature measurement - with a measuring inset, protected from the process medium by a strong thermowell, and a connection head changed little over the years and is mainly based on discoveries from the $19^{\text {th }}$ century. An important innovation was introduced in 1978 [1], with the implementation of an electronic transmitter inside the connection head. This allowed the measuring circuit and the sensor to be located close to each other, thus reducing sensitivity to electromagnetic interferences which impact sensor accuracy and introduce signal noise.

Some time ago, the temperature sensor has been transformed once more, making it autonomous by introducing wireless communication as well as an energyharvesting power supply that feeds the instrument from the temperature gradient between the process and its surroundings (Figure 1). These two technologies have been integrated into a fully autonomous temperature instrument series [2]. Their introduction was a major milestone and an enabler for wireless communication in process automation.

One remaining shortcoming of industrial temperature measurement devices, however, is the thermowell, which protects the sensitive measuring inset from the hot, chemically aggressive, abrasive or pressurized flow inside pipes, boilers and vessels (Figure 2).

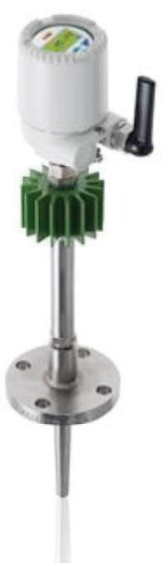

Figure 1 Autonomous temperature instrument, using the temperature difference between process and ambient for energy harvesting

Among the drawbacks of thermowells are the induced pressure drop and subsequent vortex shedding, which can result in dynamic bending stress and ultimately failure of the thermowell. Failure is especially critical for plant safety. Therefore, standards require sophisticated and often individually adapted thermowell designs. On the one hand, these can incorporate 
oversized safety margins which are detrimental for measurement accuracy and latency, on the other hand they are leading to high number of variants unwanted by manufacturers. In addition, thermowells are problematic for the process as the pressure drop also requires higher pump power consumption, and cleaning of pipes is impeded. Last but not least, the required welding is fairly expensive and retrofitting requires shutdown of the process.

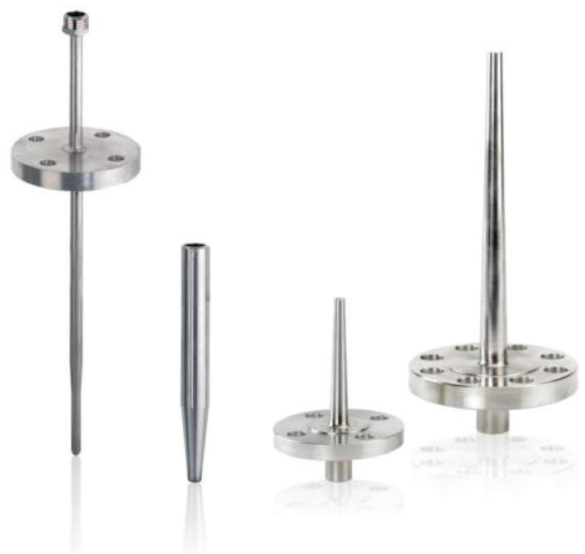

Figure 2 Thermowells typically used for heavy duty oil and gas applications

In 2010, in response to some of these challenges, ASME updated its basic standard for thermowell calculation, resulting in more robust thermowells with larger diameters, stronger materials and shorter lengths [3]. These changes merely amplified the measurement disadvantages mentioned above.

\section{Non-invasive temperature measurement}

Thermowells can be eliminated by using a noninvasive temperature measurement. Noninvasive instruments leave pipes and vessels untouched, with many advantages:

- Pipe shell is not penetrated.

- Installation does require neither process shutdown nor welding on-site

- Possibility of contamination is eliminated, both for the environment and for the process.

These advantages have considerable implications: Measurement points are now easy to install and thus can be used on a temporary basis, e.g. during set-up and test of a new process, or, if there are issues in production, for root cause analysis. As soon as a satisfactory situation has been arrived at, the number of measurement locations can be reduced to an economically and technically appropriate long-term value.
DOI 10.5162/sensoren2016/P5.5 Why has noninvasive temperature
measurement not been widely used before?

A straightforward way to obtain a noninvasive temperature measurement would be to attach an existing instrument to the surface of a pipe or vessel instead of introducing it into a thermowell. However, the temperature sensor is then further away from the process medium, so response time will be impaired and ambient influences will have a bigger influence on the measurement accuracy.

A good noninvasive temperature instrument in the process industry therefore has to have an appropriate design of the thermal pathway from the process to the sensor, which includes all materials and all interfaces through which the heat has to be transferred. It would also be beneficial if the existing (thermowell design) instrument could be adapted to fit the needs: This not only reduces the development effort significantly, but also keeps the number of variants and additional parts low.

\section{A challenging use case}

Since the easiest way described above, i.e. attaching an existing instrument to a pipe surface, would show major deviations with respect to accuracy and latency, a series of measurements has been carried out at a customer site in food \& beverage industry. It revealed a detailed picture of the thermal situation at and around the instrument as well as at the adapter that connects the instrument to the pipe. After determining the root cause of the typical measurement issues, the design of the adapter was improved and tested in multiple iterations, in close cooperation with modeling (see below). The inset was also modified to account for the altered connection to the process. In addition, the intuitively used interface material, thermal grease, was replaced by a novel solid-state material which combines high thermal conductivity with high mechanical adaptability to the adjacent surfaces. In the final configuration, measurement error was reduced to approximately $1 \mathrm{~K}$, and response time was decreased by 75 percent. As a result, both performance parameters were close to those of an invasive temperature instrument.

\section{Modeling}

Physical understanding of the measurement point and subsequent modeling and simulation of the thermal situation (see also [4]) were important for arriving at a good design. Finiteelement simulations were used to calculate temperature fields for given boundary conditions. In Figure 3, the temperature field is shown for a typical configuration.

The thermometer inset consists of a Pt100sensor element that is located in a stainless 
steel rod, together with a four-wire contact structure. The wired Pt100-structure is embedded partly in compressed Mg-oxidepowder and equipped partly with Al-oxide-parts for mechanical stability and safe wiring.

It is found that, for standard configurations, without dedicated measures, the sensor element likely sees a temperature, which is strongly influenced by ambient conditions. As shown schematically in Figure 4, in particular the inset rod tends to develop strong temperature gradients which can spoil the measurement.
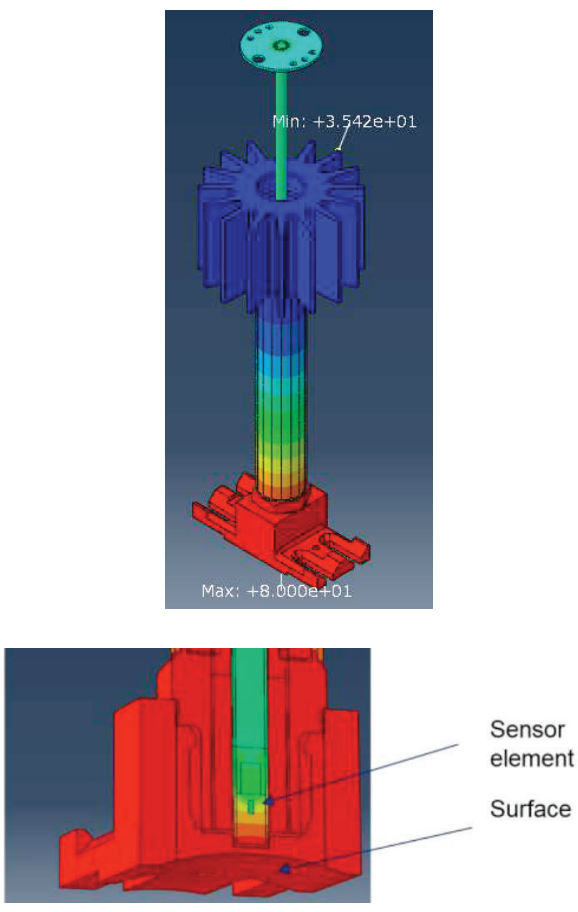

Figure 3 Finite-element result for the temperature field (in ${ }^{\circ} \mathrm{C}$ ) in a typical setup before geometry optimization

Simulations were used, then, to identify the relevant design parameters from the field behavior. Of course, a number of thermal coupling parameters or material data are only known to limited accuracy. Nevertheless, parametric studies help considerably to find bottlenecks of heat flow. For thermal design of the surface adapter and its interfaces, we investigate the temperature profile along a straight line from the pipe surface along the centerline of the inset rod. Figure 4 a) shows how the path is chosen. In Figure $4 \mathrm{~b}$ ), the temperature profiles are plotted along the path as functions of distance from the pipe surface. Discontinuities of the curve are connected to localized contact resistances between paths. A discontinuous slope of the curves indicates a change in material conductivity. For illustration, the internal setup of a standard thermometer inset is given below the $x$-axis. Depicted are the Pt100-element, an aluminum-oxide cylinder
DOI 10.5162/sensoren2016/P5.5 part and the Mg-oxide powder-filled volume, where the four copper measurement wires are embedded.

On the upper left side of Figure $4 \mathrm{~b}$ ), a pipe surface temperature of $80^{\circ} \mathrm{C}$ is imposed as boundary condition. Target of the investigation is to lift up the sensor temperature (light blue area) as close as possible to that value. The effect of design changes can be recorded in detail. A few examples are shown in Figure 5.

The potential impact of a potting is particularly obvious in this case, since it may reduce the error by more than 50 percent in this case.

a)
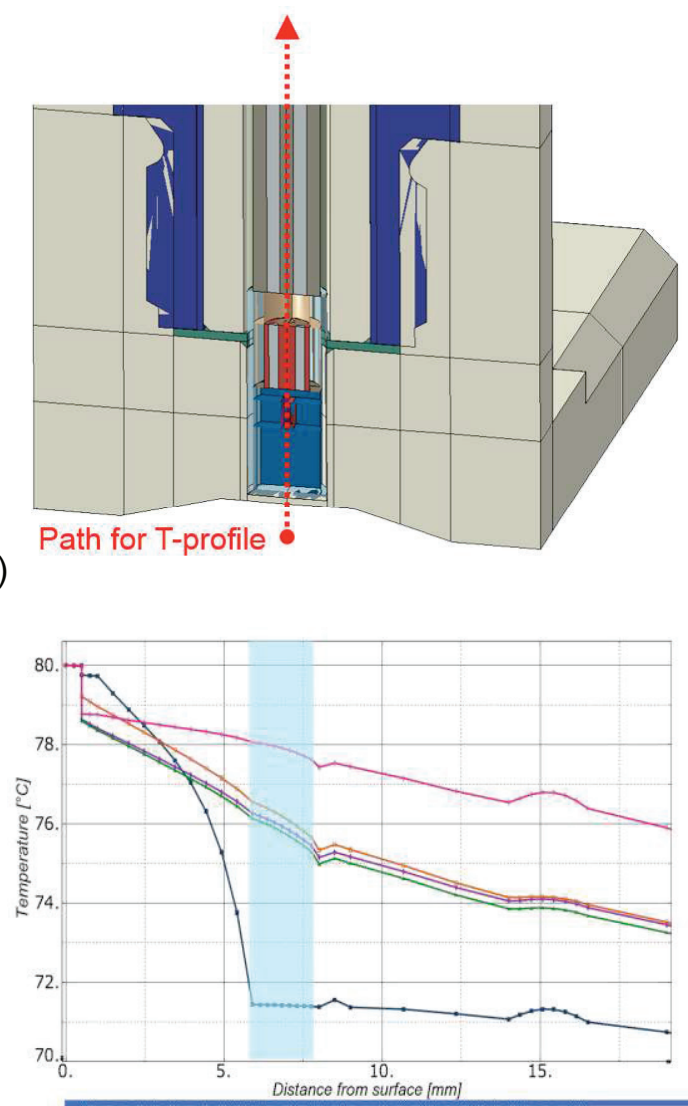

b)

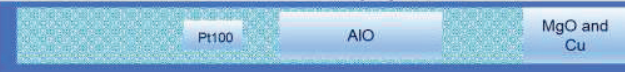

Figure 4 Comparative simulation study for relevance of design parameters.

a) Path through the device chosen for study of the temperature profile

b) Calculated temperature profiles along the path, for a variety of designs. The relevant region for the sensor element is marked blue.

\section{Easy installation}

The new adapter design can be mounted onto a wide variety of pipe diameters, only the length of the clamps (simple steel bands) has to be adjusted, thus greatly reducing the number of variants and increasing flexibility. The final design's lower complexity requires less machining and allows a particularly simple installation, which is beneficial in hard-to-reach locations. The installation does not require 
calibration or extensive parameterization. Just a standard thermal and radiation insulation, will be necessary. The fixation is designed to be long-term stable under mechanical and thermal loads as they occur in an industrial context.

Following this optimization, the customer received four new devices (one typical installation is given in Figure 8 ), and the predicted improvements in measurement accuracy and response time were confirmed.

Sensor deviation from surface temperature $\left[{ }^{\circ} \mathrm{C}\right]$

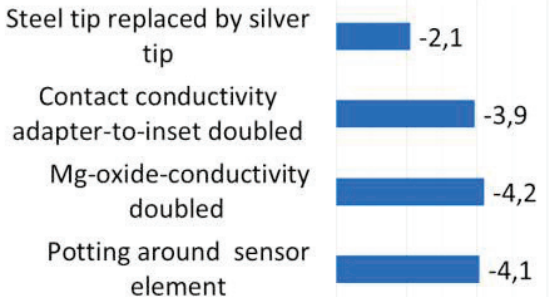

Start design

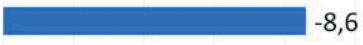

$\begin{array}{llllll}0 & -2 & -4 & -6 & -8 & -10\end{array}$

Figure 5 Influence of successive design changes on the difference of surface and sensor temperature (finite-element calculation results)

\section{Model-based measurement corrections}

Applications with extreme spatial or temporal temperature gradients pose a challenge to the complete closure of the gap between the performances of the noninvasive sensor and its invasive counterpart - both in terms of measurement accuracy as well as response time.

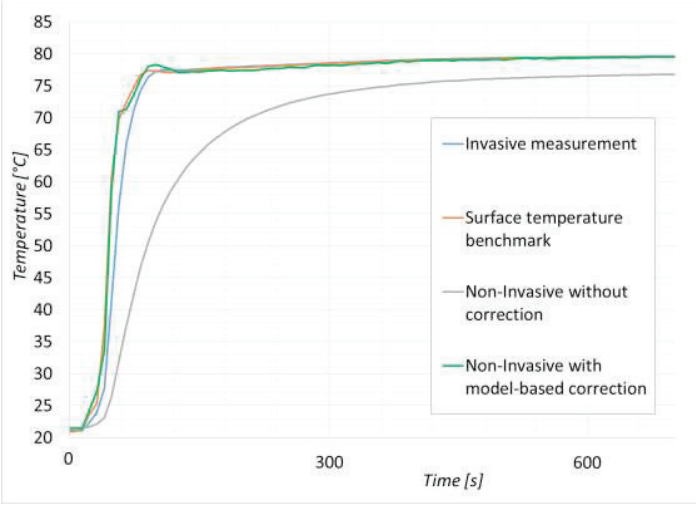

Figure 6 Response behavior of a typical uncorrected adapter structure and the result of model-based correction, as compared to an ideal surface measurement benchmark and to invasive measurement

At a certain point, for physical reasons, passive thermo-mechanical design changes will not lead to further improvement. Additional
DOI 10.5162/sensoren2016/P5.5 progress, can be made if modeling know-how is converted into reduced system representations, e.g. into thermal network models, which can efficiently be used for measurement value correction. Free parameters of the reduced model are identified by tuning to reproduce experimental results.

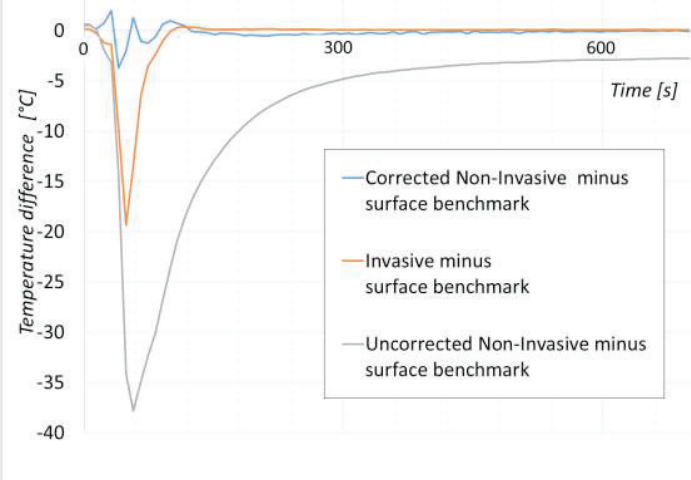

Figure 7 Deviations of the different measurement concepts from the surface temperature benchmark

Figure 6 shows the improved step response behavior of the measurement after inclusion of model-based knowledge into signal processing. The data are taken from an adapter structure which is in itself not yet optimized, in order to illustrate the strong effect of the model. In the plot shown here, an ideal surface temperature measurement has served as a benchmark, which is considerably more ambitious than matching a thermowell measurement response. One observes the well-known fact that an appropriate surfacetemperature measurement shows a quicker response than invasive sensing.

In Figure 7, residual errors are plotted for this highly dynamical situation. Obviously, the nonoptimized adapter structure leads preliminarily to a longer response time and larger errors than the invasive measurement. The modelbased correction, then, is capable to make use of the non-invasive data to match the surface temperature better than the invasive thermowell measurement.

\section{Conclusion: A new flexibility}

Non-invasive temperature measurement can match invasive in many cases. We have found that a suitable adapter structure reduces static errors to less than a Kelvin for typical flows of water-like fluids in pipes. The response time approaches that of invasive setups, in spite of the robust mechanical design, which is a necessary sensor packaging in harsh industrial environments.

For statically and dynamically challenging applications, a model-based concept has been 
demonstrated to give results that almost match those of an ideal surface sensor.

Non-invasive, wireless and energy-autonomous temperature measurements, therefore, create a remarkable new flexibility in practice. Applications that add a high value - but traditionally have been difficult to justify from a cost and time perspective - are now well within reach.

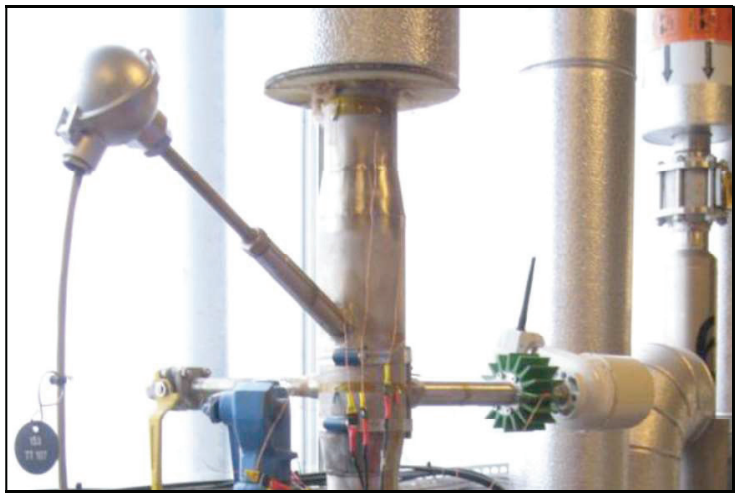

Figure 8 Pilot-installation of an autonomous and non-invasive temperature device, before insulation, close to a standard thermowell installation for benchmark purposes.

One good example of such an application is short-term instrumentation of processes during optimization and continuous improvement exercises or energy efficiency initiatives. Another example is to supply a heat exchanger asset monitor - a condition monitoring tool that identifies heat exchanger performance changes and operational degradation - with the temperature inputs it requires to guarantee more energy-efficient operation and reduced maintenance cost. In large facilities, improved heat exchanger performance delivers substantial energy savings.

\section{References}

[1] Industrial temperature measurement, basics and practice, Handbook for customers, ABB Automation Products, (2008).

[2] M. Ulrich et al., "Autonomous wireless sensors for process instrumentation," in GMA / ITG - Fachtagung: Sensoren und Messsysteme 2012, Nuremberg.

[3] Thermowells, ASME standard no. PTC 19.3 TW-2010.

[4] J. Gebhardt and K. König, "Model-based development for an energy-autonomous temperature sensor," in VDI/VDE Mechatronik 2013, Aachen, Germany, 2013, pp. 177-181. 